\title{
Orientation émotionnelle mathématique : la raison esthétique
}

\author{
Henri Volken
}

\section{(2) OpenEdition}

Journals

Édition électronique

URL : http://journals.openedition.org/ress/72

DOI : $10.4000 /$ ress. 72

ISSN : 1663-4446

Éditeur

Librairie Droz

Édition imprimée

Date de publication : 1 mai 2009

Pagination : 121-134

ISBN : 978-2-600-01303-1

ISSN : 0048-8046

\section{Référence électronique}

Henri Volken, "Orientation émotionnelle mathématique : la raison esthétique », Revue européenne des sciences sociales [En ligne], XLVII-144 | 2009, mis en ligne le 01 mai 2012, consulté le 01 mai 2019.

URL : http://journals.openedition.org/ress/72 ; DOI : 10.4000/ress.72 


\section{Henri VOLKEN}

\section{ORIENTATION ÉMOTIONNELLE MATHÉMATIQUE: LA RAISON ESTHÉTIQUE}

Dans un classement populaire des sciences rationnelles, les mathématiques occuperaient probablement l'une des premières places, avec en prime la mention «raison froide et activité solitaire ». Et le préjugé très répandu d'une science où les émotions ne jouent aucun rôle, accompagnerait le plus souvent toute évocation de ce classement. Et puisque les émotions sont absentes, l'activité ne peut pas être humaine. Ce qui, entre parenthèses, reflète l'idée courante, mais un peu paradoxale, que ce sont les émotions qui nous rendent humains, alors que la raison nous rapprocherait plutôt de la machine. Car ce qui nous distingue essentiellement des animaux - qui eux aussi ont des émotions, comme la peur - c'est précisément l'existence chez l'homme des régions associatives du néo-cortex, qui a rendu possible l'émergence de la raison. Ne serait-ce donc pas plutôt la raison qui est le signe distinctif de l'humain?

Mais cela n'est qu'un aspect annexe de la question qui nous intéresse ici: quelle est la part de l'émotion - plus généralement de l'affectif - dans la démarche du mathématicien? Est-ce raisonnable, et même possible, de séparer une activité faisant intervenir la raison, de toute forme de mouvement affectif, en particulier de toute émotion? Nous allons essayer de montrer que bien sûr cela est impossible, rejoignant par-là des courants de pensée divers qui ont surgi depuis une trentaine d'années. Des exemples célèbres, comme l' «Erreur de Descartes» de Damasio, ont popularisé ce thème dans un cercle très large. Ce qui nous intéresse ici avant tout, c'est de porter un regard plus spécifique sur l'activité du mathématicien, qu'il soit enseignant ou chercheur de pointe. Et d'y trouver des lieux où une certaine forme d'émotion est indissociable de la pensée mathématique.

Revenons un instant sur l'expression «raison froide et activité solitaire ». Si par «froid» on suggère l'absence d'émotion, alors cette expression ne caractérise pas la démarche du mathématicien. Il y a au contraire de nombreux moments affectifs qui stimulent, influencent et parfois initient des mouvements créatifs ou simplement calculatoires dans son activité. D'autre part sa démarche n'a rien de solitaire et suppose de plus, comme nous allons le montrer, une composante sociale très développée, composante qui donne cette remarquable unité aux mathématiques, qui à son tour leur confère le prestige certain d'une science cumulative.

Pourquoi porter alors un regard négatif sur les mathématiques? Quelques éléments de réponse s'imposent rapidement:

- les objets mathématiques n'ont pas de «réalité», (sauf pour les platoniciens)

- les vérités mathématiques s'expriment souvent dans une langue hautement formalisée et largement incompréhensible pour un grand public 
- un nombre important de résultats fondamentaux ne peuvent pas se traduire en termes simples et communément accessibles

- l'aspect émotionnel semble absent et même exclu par un formalisme strict

- la composante sociale du travail mathématique n'apparaît pas clairement

Mais paradoxalement, si les émotions semblent absentes du discours sur et autour des mathématiques, celui-ci est souvent teinté d'une certaine passion: des exclamations comme «J'ai toujours détesté les mathématiques», "Les mathématiques sont une torture », et plus rarement: «J'adore les mathématiques » et ainsi de suite, sont courantes.

Si le langage mathématique lui-même paraît masquer les émotions sousjacentes à leur établissement, c'est que les résultats mathématiques s'expriment en général de manière concise, formelle, effaçant les traces de leur genèse. Mais ce goût pour le minimal indique déjà l'une des directions privilégiées dans laquelle s'exprime l'aspect affectif du travail mathématique: l'émotion esthétique. Les sciences formelles sont fortement influencées par l'idée du beau. Quelques citations célèbres peuvent illustrer l'importance que les mathématiciens accordent à cet aspect de leur domaine.

Par exemple:

Les sciences mathématiques en particulier mettent en évidence l'ordre, la symétrie et la limitation; et cela ce sont les grandes formes de la beauté

Ou encore:

Aristote

The mathematician's patterns, like the painter's or the poet's must be beautiful; the ideas, like the colours or the words must fit together in a harmonious way. Beauty is the first test: there is no permanent place in this world for ugly mathematics

G. H. Hardy

Et finalement:

Alors, quelles sont les entités mathématiques auxquelles on attribue le caractère de beauté et d'élégance, et qui sont capables de développer en nous une sorte d'émotion esthétique? Elles sont celles dont les éléments sont disposés harmonieusement de façon que l'esprit puisse sans effort en embrasser la totalité tout en réalisant leur détails

...les combinaisons utiles sont précisément les plus belles

H. Poincaré

En réalité de nombreux autres mathématiciens et philosophes se sont exprimés dans ce sens, depuis Platon en passant par Galilée, Leibniz, Newton jusqu'au vingtième siècle, avec Hadamard et Russell notamment. Il est même question dans la communauté des mathématiciens de se demander quelle est la «plus belle formule mathématique». L'un des candidats sérieux est la célèbre formule d'Euler:

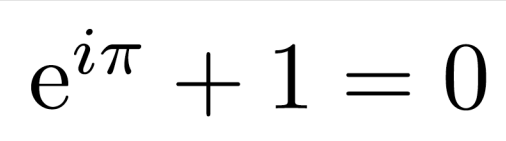


D'où vient l'attrait évident de cette formule? Non seulement de sa concision et simplicité, c'est le cas pour de nombreuses formules, mais du fait qu'elle lie aussi clairement et simplement cinq concepts importants dans le développement des mathématiques:

- le zéro, dont l'introduction au XIIe siècle dans notre culture a rencontré une forte résistance

- le un, l'unité arithmétique qui, par l'addition, permet de reconstruire chaque nombre

- l'unité imaginaire $i$, introduite par des mathématiciens italiens au $\mathrm{XVI}^{\mathrm{e}}$ siècle et qui est à l'origine de la construction des nombres complexes

- le nombre $\pi$, le rapport entre circonférence et diamètre d'un cercle

- le nombre $e$, nombre irrationnel qui est la base des logarithmes naturels

La beauté de la formule provient de l'intégration de ces cinq concepts très différents et importants pour l'histoire des mathématiques, dans un objet formel compact et d'une simplicité lumineuse. L'émotion esthétique naît ici d'une parfaite adéquation entre la forme et la densité d'information qu'elle véhicule.

C'est cette insistance explicite sur la beauté dans le discours des scientifiques qui a guidé notre choix de prendre l'émotion esthétique comme principal exemple de l'irruption d'un mouvement affectif dans le territoire - supposé pur et froid de la connaissance mathématique.

Le mathématicien Paul Erdös utilisait souvent l'expression «Le Grand Livre» (The Book) pour désigner l'ouvrage dans lequel, selon sa métaphore, Dieu inscrivait les preuves parfaites des théorèmes mathématiques ${ }^{1}$. Cela montre bien que chez certains mathématiciens le beau peut être un argument presque aussi important que le $v \mathrm{rai}^{2}$. Mais quelle est l'influence de cette émotion esthétique sur leur démarche? Nous allons essayer de montrer non seulement que cette influence est multiple mais que le plaisir et la recherche du beau n'est pas la seule émotion à jouer un rôle important dans le travail mathématique. Pour cela nous allons élargir le cadre de notre présentation en introduisant un concept général proposé par Maturana $^{3}$ en 1988, que nous allons tenter d'adapter à la réalité mathématique dans le passage qui suit.

1 Une première approximation, imparfaite bien sûr, de ce «Grand Livre», est parue en 1998, et en 2001 pour la traduction française sous le titre de «Raisonnements divins» par M. Aigner \& G. M. Ziegler, Springer

2 On répliquera avec quelque raison que tout cela est une vision idéale du monde mathématique, et décrit une démarche élitiste, probablement loin des préoccupations d'applications mathématiques plus instrumentales

3 Maturana,H. R. (1988): Reality: The search for objectivity or the quest for a compelling argument, Irish Journal of Psychology, 9, 25-82 


\title{
I. L'ORIENTATION ÉMOTIONNELLE MATHÉMATIQUE
}

Dans son article Maturana introduit le terme d'orientation émotionnelle - ou parfois simplement culture - pour décrire le cadre global des préférences partagées par les chercheurs d'un domaine. Cette approche, marquée par l'idée de connaissance incorporée, semble être particulièrement appropriée pour la description de la démarche mathématique ${ }^{4}$. Elle nous permettra d'en traiter simultanément les aspects affectifs, les aspects purement cognitifs ainsi que les aspects sociaux.

L'introduction du concept d'orientation émotionnelle se fait chez Maturana essentiellement selon les étapes suivantes ${ }^{5}$ : le domaine d'explications choisi par un observateur dépend du critère de validation que celui-ci accepte pour qu'une re-formulation donnée de la réalité soit une explication. Ensuite chaque domaine d'explications constitue aussi un domaine d'actions considérées comme légitimes, car soutenues par les explications de ce domaine. Comme chaque domaine d'actions légitimes est un domaine de cognition, chaque domaine d'explications est un domaine de cognition. Finalement, qu'un observateur opère dans un domaine d'explications ou un autre, dépend de sa préférence (émotion de l'acceptation) pour les prémices de base de ce domaine ${ }^{6}$.

Je cite dans la forme originale le passage suivant du même article qui introduit le concept d'orientation émotionnelle:

\begin{abstract}
The operations that constitute the criterion of validation of scientific explanations are the same that we use in the operational validation of the praxis of our daily life as human beings. It follows from this that, in a strict operational sense, what distinguishes an observer in daily life from an observer as a scientist is the scientist's emotional orientation to explaining his [...] consistency in using only the criterion of validation of scientific explanations for the system of explanations that he [...] generates in his [...] particular domain of explanatory concerns, and his [...] commitment to avoid confusing phenomenal domains in his $[. .$.$] generation of scientific explanations.$
\end{abstract}

Le cadre tracé par Maturana pour le concept d'orientation émotionnelle permet d'englober les nombreuses facettes du travail du mathématicien. On peut définir l'orientation émotionnelle, dans le domaine des mathématiques, comme le partage de savoirs et de croyances, de préférences en ce qui concerne les conventions, formalisations et principes déductifs, aussi bien que des moyens de communiquer et d'agir. Plus précisément on peut distinguer en général trois dimensions dans l'orientation émotionnelle ${ }^{7}$ :

- Critères pour l'acceptation des explications

- Cohérences opérationnelles structurant les explications

- Actions considérées comme légitimes

\footnotetext{
4 Cf Drodge \& Reid dans: Mathematical Thinking and Learning, 2: 4, 249-267, 2000

5 Voir Maturana, op. cité. J'ai essayé de respecter dans ce bref résumé au mieux le point de vue ainsi que la terminologie de Maturana

6 Et non pas de considérations purement rationnelles

7 Suivant en cela Mingers, J. (1995), Self-producing systems: Implications and applications of autopoiesis, New York
} 
Ce qui détermine l'adhésion à un domaine de connaissance c'est essentiellement le type d'explications que le chercheur est disposé à accepter, à utiliser dans son travail et à échanger avec ses pairs. Ce choix ne se fait pas purement rationnellement, mais dépend de facteurs qui peuvent être d'ordre affectif, faisant souvent appel à une émotion esthétique. Le choix est dicté par des affinités avec les prémices de base qui constituent le fondement du domaine. Ces affinités concernent la plupart du temps l'élégance, la clarté, la simplicité ou la concision de la formulation des prémices. Mais le contexte social joue également un rôle important en favorisant les aspects de notoriété, voire de mode. Ce dernier facteur peut, de part sa nature, varier fortement dans le temps.

Le deuxième thème proposé par Maturana, le domaine des cohérences opérationnelles, décrit les façons caractéristiques de penser, de parler et d'agir dans une communauté, ici la communauté des mathématiciens. Il concerne au moins quatre axes distincts, que l'on peut désigner schématiquement par langage, émotions, actions et cognition.

Le langage des mathématiques est fortement marqué par le choix du formalisme et des notations. A de nombreuses reprises l'histoire des mathématiques a été influencée par l'utilisation d'une notation plus simple ou plus opérationnelle. Il suffit pour le voir de tenter une opération sur des nombres en utilisant la notation romaine. C'est le caractère positionnel de l'écriture arabe qui a permis le développement de l'arithmétique et par là de l'analyse et de la physique. Et l'analyse précisément s'est développée grâce à la notation de Leibniz, qui à l'origine était en concurrence avec celle de Newton. De même c'est à la notation de Peano que la logique formelle doit son essor au cours du $\mathrm{XX}^{\mathrm{e}}$ siècle.

L'un des moteurs essentiels de la découverte - et du travail - mathématique est d'ordre émotionnel. Les chercheurs évoquent très souvent - nous en avons vu quelques exemples célèbres - le plaisir esthétique dans leur démarche: beauté d'une démonstration, élégance d'une construction ou harmonie d'une argumentation sont souvent évoquées. Mais la plupart du temps c'est de la joie de la découverte de la vérité qu'il est question, bien que cela soit probablement le propre de toute science. Encore que les mathématiciens aient une vision très précise - peutêtre plus limitée il est vrai - de la vérité en l'associant, comme nous allons le montrer plus loin, au concept de cohérence. Une émotion souvent exprimée concerne enfin l'idée de complétude, souci ubiquitaire en mathématiques.

Les cohérences opérationnelles comprennent aussi les moyens d'actions d'une communauté. Les questions ici sont les formes d'échange d'information, en particulier de résultats nouveaux, la manière dont sont produites les confrontations de preuves et comment les acceptations et les réfutations s'expriment, finalement comment se traduisent ces opérations socialement.

Du point de vue de la cognition, la perspective mathématique demande un mélange original d'intuition, d'imagination - parfois un brin de folie - avec un sens aigu de rigueur déductive. Cela parce que les mathématiques sont une science largement cumulative, donc dépendante du corpus accumulé et de la découverte de la nouveauté. La mémoire collective de ce domaine doit présenter un équilibre délicat entre plasticité et stabilité. 
Pour le chercheur, partager l'orientation émotionnelle des mathématiciens, signifie entre autres les points suivants. Il doit admettre la «réalité » des objets mathématiques sous l'une des formes possibles. Il peut adopter - entre autres, mais ce sont peut-être les orientations principales - soit la position formaliste, c'est-à-dire que pour lui les mathématiques sont une simple manipulation de symboles, soit la position platonicienne, dans laquelle il admet l'existence des objets mathématiques, même si cette existence ne se situe pas dans notre monde matériel. Il doit croire en une logique déductive partagée par ses pairs et agir selon ses règles. Cette logique n'est d'ailleurs pas forcément explicite ou formalisée, mais elle doit correspondre aux actions considérées comme légitimes dans ce domaine du savoir. D'autre part le mathématicien doit avoir la capacité de reconnaître, de rejeter ou d'accepter des conjectures et des preuves, mais aussi d'en produire de nouvelles. Cela n'est possible que s'il adhère au langage et aux présupposés communs. Finalement, il préférera les constructions, démonstrations et résultats «esthétiques», même si la définition de ce concept peut sembler quelque peu floue.

Drodge et Reid (op. cité) rappellent l'une des composantes affectives de l'orientation émotionnelle mathématique: les qualités morales du mathématicien (et plus généralement du scientifique). Il s'agit ici de l'attitude du mathématicien concernant l'acceptation et le rejet de conjectures dans le processus global de l'explication scientifique. G. Polya ${ }^{8}$ formule ses exigences de la manière suivante: la première est celle du courage intellectuel, c'est-à-dire la faculté de produire des conjectures qui peuvent remettre en question son savoir établi. La deuxième est l'honnêteté intellectuelle, la volonté de réviser ses croyances en face d'une contradiction. Et finalement ce que Polya appelle la sage retenue ${ }^{9}$ qui consiste à rejeter certaines formes de résistance, comme l'intimidation, à une conjecture nouvelle.

\section{LA RAISON ESTHÉTIQUE}

Nous avons déjà évoqué différents énoncés de mathématiciens qui soulignent le rôle essentiel joué par les considérations esthétiques dans la démarche mathématique, et cela non seulement dans l'appréciation de la beauté d'une preuve ou d'une construction, mais également dans la phase heuristique de la recherche. Nous avons cité Poincaré et Hardy, on pourrait citer l'exemple de Hadamard ${ }^{10}$, Penrose, Hofstadter et bien d'autres. Comme l'aspect esthétique constitue une part importante du monde affectif, plus particulièrement émotionnel, du mathématicien, nous allons essayer d'en préciser les axes principaux en décrivant tout d'abord quelques rôles dans lesquels apparaît ici l'esthétique, puis les concepts primitifs qui expliquent son importance pour le mathématicien.

Parmi les rôles que l'esthétique joue dans la démarche mathématique on peut en distinguer au moins quatre qui semblent fondamentaux: les rôles évaluatif, génératif, motivationnel et sélectif. Ces rôles ne peuvent pas être rigoureusement distincts, mais pour l'essentiel ils sont caractérisés selon les points suivants.

\footnotetext{
8 G. Polya (1954), Mathematics and plausible reasoning, Princeton 1954

9 «Wise restraint» chez Polya
} 
Comment s'y retrouver parmi les innombrables résultats (théorèmes, nouvelles démonstrations) que les mathématiciens produisent chaque année? Lequel de ces résultats présente un intérêt suffisant pour capter notre attention? Il n'y a pas véritablement de critère interne qui permette un tel choix. Et c'est là que l'on peut situer le rôle évaluatif de l'esthétique en mathématique ${ }^{11}$. Ce rôle possède deux facettes. D'une part il facilite une sélection des objets importants, sélection partagée par une partie de la communauté. Cette sélection se trouve de ce fait renforcée et encouragée. Mais le rôle évaluatif ne s'arrête pas là. Il détermine aussi la manière de formuler et d'exprimer les résultats et de les communiquer. Cette double fonction permet à la composante esthétique d'influencer non seulement la structure, mais également la dynamique du savoir mathématique.

La citation de Poincaré, que nous avons évoquée plus haut, nous donne une première indication sur le rôle génératif que la quête du «beau» peut jouer. Toute une partie de la recherche en mathématiques se situe à un niveau subconscient et fait appel, non pas à la déduction pure, mais à l'abduction et l'invention, et privilégie l'imagination et l'intuition. Et l'esthétique prend alors la fonction de guide dans ces modes non propositionnels de raisonnement. Elle favorise la génération de nouvelles idées, perspectives et démarches, impossibles par la logique seule. La considération de mondes possibles en même temps que la sélection, parmi ces mondes, des plus prometteurs, sont les caractéristiques du rôle génératif de l'esthétique, même si ce rôle est difficile à définir avec précision. Mais le concept capte en plus un aspect omniprésent en mathématiques, l'idée de jeu. Que les mathématiques comportent une composante ludique, semble indéniable. Et c'est elle qui, alliée à la recherche du beau, peut parfois ouvrir des perspectives toutes nouvelles vers des solutions imprévues ${ }^{12}$.

Qu'est-ce qui peut motiver un chercheur à se lancer dans des recherches en mathématiques et à persévérer dans cette direction? Les raisons qui pourraient l'en dissuader sont nombreuses. Tout d'abord le lien avec la réalité n'est pas très clair, sinon totalement absent. Ensuite la démarche est réputée ardue et solitaire. Et la considération sociale reste faible, même si tout le monde s'accorde pour trouver cette voie difficile. La raison de ce choix peut à nouveau être rapprochée d'une préoccupation esthétique. Si la difficulté en elle-même explique pour certaines personnes l'attrait d'un problème mathématique, il faut probablement chercher dans l'idée de surprise, de paradoxe ou simplement d'ordre, la clé de cette fascination. La surprise, qui remet en question certaines attentes du chercheur et le paradoxe qui remet en question ses certitudes, participent indirectement, par les défis qu'ils représentent, d'une motivation de type esthétique. Nous allons essayer plus loin de compléter cette liste par d'autres propriétés participant du même mouvement de motivation.

Les mathématiques sont devenues, en particulier dans le courant du $\mathrm{XX}^{\mathrm{e}}$ siècle, un domaine du savoir très étendu. Les théorèmes, preuves et constructions qui

${ }^{10}$ The mathematician's mind; The psychology of invention in the mathematical field, Princeton, 1945

${ }^{11}$ Voir par exemple: A. M. White, Essays in humanistic mathematics, Washington D.C., 1993

${ }^{12}$ Silver, E. \& Metzger, W. (1989), Aesthetic influences on expert mathematical problem solving, In D. B. McLeod \& V. M. Adams (Eds), Affect and mathematical problem solving, New York 
paraissent chaque année forment une quantité d'information énorme et une bonne partie de ces résultats disparaissent sans laisser de trace dans l'accumulation des connaissances mathématiques. Comment sont sélectionnés les éléments qui resteront? Parmi tous les critères qui peuvent intervenir - modes, nouvelles applications technologiques, politique de recherche - c'est encore une fois l'aspect esthétique qui tient une part importante du rôle sélectif. Il ne s'agit pas exclusivement d'esthétique au sens étroit, car définir ce qui est beau peut dépendre du contexte social, notamment de la reconnaissance qu'on peut attendre pour la découverte de résultats nouveaux. Néanmoins un grand nombre de facteurs comme l'attrait de l'objet géométrique, la concision d'une démonstration ou même une rigoureuse économie formelle, peuvent infléchir la sélection d'un domaine ou d'une problématique particulière.

Après ce bref aperçu des quatre rôles que l'esthétique joue dans la démarche mathématique, nous allons nous pencher sur quelques concepts-clé souvent évoqués à ce propos dans les commentaires que les mathématiciens font sur leur expérience personnelle. Comme nous allons le voir, ces concepts ne sont pas indépendants, mais forment un réseau qui les lie de multiples manières. Les paragraphes qui suivent tentent de décrire quelques aspects de ce réseau.

L'une de ces notions, la vérité semble jouer un rôle particulièrement important puisque c'est à sa découverte que paraissent tendre tous les efforts. Un résultat faux n'a pas d'attrait et apparaît comme « laid». Mais de quelle vérité s'agit-il puisque les mathématiques n'ont pas de «réalité»?

Il est donc difficile de dire que la vérité est une adéquation avec le monde. On peut avoir cette illusion en pensant à nos expériences de tous les jours dans lesquelles nous sommes amenés à additionner deux nombres. Supposons que nous voulions rajouter deux livres aux trois qui se trouvent déjà sur la table. Le cinq s'impose comme solution du problème deux plus trois par simple inspection des objets que nous sommes en train de manipuler. Nous avons l'impression de pouvoir vérifier ce résultat en comptant ceux-ci et sommes convaincus de sa généralité. Mais qu'en est-il lorsqu'il s'agit d'additionner deux millions et trois millions? Une vérification pratique semble exclue alors que le résultat ne fait pas de doute.

Dans le cours de leur histoire, les mathématiques se sont progressivement émancipées de préoccupations pratiques. Une abstraction certaine et une formalisation de plus en plus rigoureuse les ont poussé à se rapprocher d'une vérité-cohérence. L'utilisation de la logique et de ses possibilités de valider une argumentation a permis de remplacer d'une certaine façon l'ontologie par des considérations de cohérence: Ce qui n'est pas contradictoire existe. D'où la liberté d'explorer des «mondes possibles» comme les nombres imaginaires, les géométries non euclidiennes ou les logiques non aristotéliciennes, pour lesquels il n'existe pas de véritable sémantique «réaliste», mais uniquement des sémantiques formelles. Ce plaisir de ne pas être limité par ce qui existe, mais d'imaginer ce qui pourrait être, tout en utilisant les mêmes outils, participe d'un mouvement qu'on peut rapprocher sans hésitation d'une émotion esthétique.

Ce deuxième concept-clé, la cohérence, donne son sens à la démarche mathématique. Comme l'ordre dans la nature, la cohérence est une exception dans les grands ensembles d'énoncés. Il lui est par conséquent attaché une aura de rareté, de beauté. La non-contradiction, particulièrement dans sa phase de recherche, est 
une motivation particulière pour le chercheur. C'est elle qui lui donne ce sentiment de complétude et d'universalité et qui permet d'utiliser un concept rigoureux de «preuve» qui à son tour favorisera les échanges sur une base partagée. Encore faut-il que la cohérence apparaisse sous une forme adéquate.

Cette forme peut être caractérisée par différents termes. L'un des plus cités par les mathématiciens est la simplicité qui possède un attrait esthétique évident pour le chercheur. On peut comprendre en partie ce goût de la simplicité par des considérations pratiques. Elle lui donne une force opérationnelle plus grande - il suffit de penser à l'importance de la notation et la facilité de sa manipulation. Elle rend également la cohérence plus visible en allégeant l'argumentation. Et finalement, les preuves et les constructions simples laissent un plus grand espoir de généralisation, tout en favorisant la communication des résultats.

La simplicité est le pendant d'une autre exigence récurrente des mathématiques: la complétude. Il s'agit du désir de trouver la portée la plus large possible pour chaque théorie, et de se rapprocher de l'efficacité, l'élégance, d'un système sans exceptions. En géométrie élémentaire par exemple, deux droites se coupent en un point, sauf si elles sont parallèles. Or la géométrie projective, qui introduit le concept de «point et droite à l'infini », élimine cette exception, et dans cette théorie deux droites distinctes ont toujours un point en commun - ultime coquetterie épistémologique. De même le développement de la science des nombres est caractérisé par des tentatives d'atteindre à une plus grande complétude: comment intégrer les nombres négatifs, fractionnaires, réels et imaginaires dans la théorie existante. Ce concept de complétude est toujours présent, fût-ce de manière diffuse, dans l'idée même de «système axiomatique». Et c'est l'une des lettres de noblesse de la logique du premier ordre d'être «complète» dans le sens d'une parfaite adéquation entre syntaxe et sémantique. Ce résultat, dû à Gödel ${ }^{13}$, est un véritable plaisir esthétique pour tout logicien!

Des tests psychologiques ont confirmé à de nombreuses reprises l'existence d'un autre lien entre esthétique et monde mathématique, connu depuis l'antiquité: la symétrie. Ainsi un visage ou un corps qualifié majoritairement de beau, possède le plus souvent la régularité et l'harmonie justement octroyées par cet effet miroir. Pour le mathématicien, loin d'être de nature statique, la symétrie est un concept essentiellement dynamique. Elle reflète pour lui l'immunité d'une structure par rapport à des transformations auxquelles elle est soumise. Une frise par exemple est insensible à certaines translations, de même qu'un pavage ou une mosaïque périodique. D'autres structures sont invariantes par rapport à des rotations ou des symétries axiales. C'est la «beauté» de ces structures qui est à l'origine de la théorie des groupes, un champ central des mathématiques. La symétrie peut se manifester dans l'espace et dans le temps, elle peut aussi concerner des objets non géométriques comme des propriétés, voire des règles. C'est toujours l'existence simultanée de transformations et d'une certaine immunité qui résiste à celles-ci, qui fait tout l'intérêt de la symétrie et aussi sa force dans la pratique mathématique. On peut rappeler à ce propos également l'importance de la symétrie dans l'évolution naturelle et le développement des êtres vivants.

\footnotetext{
13 A qui l'on doit d'ailleurs également un théorème - plus connu encore - d'incomplétude !
} 
Si la symétrie est considérée comme génératrice du beau par les mathématiciens, l'élégance est parmi les premiers qualificatifs que ceux-ci utilisent pour exprimer leur admiration pour une démonstration ou un argument ${ }^{14}$. C'est elle qui rend la fonctionnalité plus apparente en privilégiant une démarche minimale, concise et lisible. Par son souci esthétique elle favorise la pensée fluide, non propositionnelle et les associations non linguistiques. L'analogie, moteur capital dans la pensée mathématique, s'en trouve facilitée. Sans oublier que l'élégance constitue un outil important dans les échanges puisqu'elle permet plus facilement de communiquer et de convaincre.

Or très souvent les mathématiciens évoquent la concision comme l'une des composantes de l'élégance. En particulier une preuve se doit à leurs yeux d'être la plus économe possible, aussi bien dans son extension que dans le nombre de concepts utilisés. La notation doit également être spartiate et opérationnelle. Et effectivement la concision donne une plus grande visibilité aux structures décrites et permet d'entrer plus facilement dans les dialogues entre domaines voisins. Finalement, elle peut favoriser les tentatives de généralisation par une meilleure lisibilité des arguments.

Néanmoins, ni élégance, ni concision ne peuvent se passer de la rigueur, propre aux mathématiques. C'est elle qui contribue à donner à l'orientation mathématique son aspect caractéristique, fait d'un mélange d'imagination, de logique et de surprise. Mais, tout aussi important, cette rigueur doit être acceptée par les membres du même domaine et représente donc une fonction sociale importante: une science mathématique «privée» qui ne partage pas le standard de logique d'un groupe, n'a plus beaucoup d'intérêt. Au point de vue de la communication, la rigueur facilite l'exportation de définitions de raisonnements et de preuves formelles et permet la comparabilité et la décision en cas de divergence.

Mais toute la rigueur des mathématiques ne suffit pas à expliquer leur applicabilité dans le monde réel, qui pourrait bien être la conséquence de l'utilisation fréquente de l'analogie, ce processus indispensable à la reconnaissance et à l'exploitation de similitudes. On peut rapprocher l'analogie de la symétrie en étendant cette dernière à la validité d'une proposition soumise à des transformations. Si dans l'énoncé «Jupiter tourne autour du soleil sur une orbite elliptique» on remplace «Jupiter» par «Vénus», la proposition reste valide, elle est robuste par rapport à la transformation proposée. c'est donc un cas de symétrie. Or une symétrie peut être parfaite - ce qui n'est peut-être le cas que dans le monde mathématique - ou approximative. C'est dans cette dernière circonstance, celle qui représente une immunité partielle, qu'on peut parler d'analogie. Si l'analogie a une place prépondérante dans l'orientation émotionnelle mathématique, c'est qu'elle crée, de manière non déductive, des passerelles entre des domaines différents et rend éventuellement possible la découverte d'informations contenues dans la différence. L'analogie se présente donc comme un outil indispensable pour la transposition de concepts d'un domaine à un autre.

Le besoin de tester ses concepts dans des environnements nouveaux est particulièrement important pour le mathématicien. Car si l'on considère les mathéma-

${ }^{14}$ Etymologiquement élégant possède aussi la signification «qui sait choisir» 
tiques comme l'étude des structures en général, elles ont clairement une vocation transdisciplinaire. Le problème de l'exportabilité des notions devient primordial dans cette optique. D'où une nouvelle explication de l'engouement des mathématiciens pour le «beau» au sens de concis et rigoureux. Car la simplicité et la cohérence du vocabulaire, de la notation, et plus généralement du formalisme, facilite beaucoup les possibilités d'exporter le savoir mathématique. Et les échanges de notions et de contextes qui en résultent sont un stimulant précieux pour la recherche.

Les preuves formelles, mais aussi les théories, sont comme des pierres entraînées par un fleuve - par les échanges ils se polissent, se simplifient et deviennent plus élégantes. Ce qui à son tour favorise leur communicabilité, condition essentielle à la diffusion et confrontation des idées. Cet aspect social du travail du mathématicien est évident.

Mais peut-être que la véritable vocation du transfert des concepts est la recherche d'une certaine universalité des résultats. Celle-ci pourrait expliquer, partiellement en tous cas, l'applicabilité des mathématiques dans les théories du monde «réel». Et elle constituerait une sorte de «revanche» sur la non-existence d'un terrain concret des objets formels: les mathématiques ne possèdent rien en propre mais parlent de tout, ce qui est le sens de l'expression «science ou théorie des structures ». La réalité des mathématiques est distribuée sur différents mondes possibles. Le savoir mathématique ne s'applique donc pas uniquement au monde «réel».

Les concepts que nous venons d'évoquer, sous-tendent et illustrent la raison esthétique, telle qu'elle apparaît chez les mathématiciens, et qui est une composante essentielle de leur orientation émotionnelle. En les décrivant brièvement en quelques phrases, on s'aperçoit qu'ils sont intriqués et forment un entrelacement dense, un complexe si l'on se réfère au sens étymologique de ce terme. Cela explique une certaine forme de redondance présente dans l'évocation de ces notions. Imitons un instant les mathématiciens et considérons les concepts en question comme des points dans un espace abstrait. Cela nous permet de visualiser la géométrie de la situation. Les concepts eux-mêmes constituent les sommets, et les arêtes qui les relient indiquent la présence commune de deux concepts dans un contexte particulier. On peut ainsi distinguer trois «facettes ${ }^{15}$ de l'orientation émotionnelle en se focalisant sur les aspects «esthétique», «opérationnel» et «social». Ces facettes correspondent à des objets géométriques d'une certaine dimension dans l'espace des concepts choisis. La dimension étant ici le nombre de sommets - de concepts - moins un. Par exemple, la facette «esthétique» est de dimension cinq et peut être représentée schématiquement par le graphe suivant:

15 Trois simplexes dans le langage des mathématiciens 


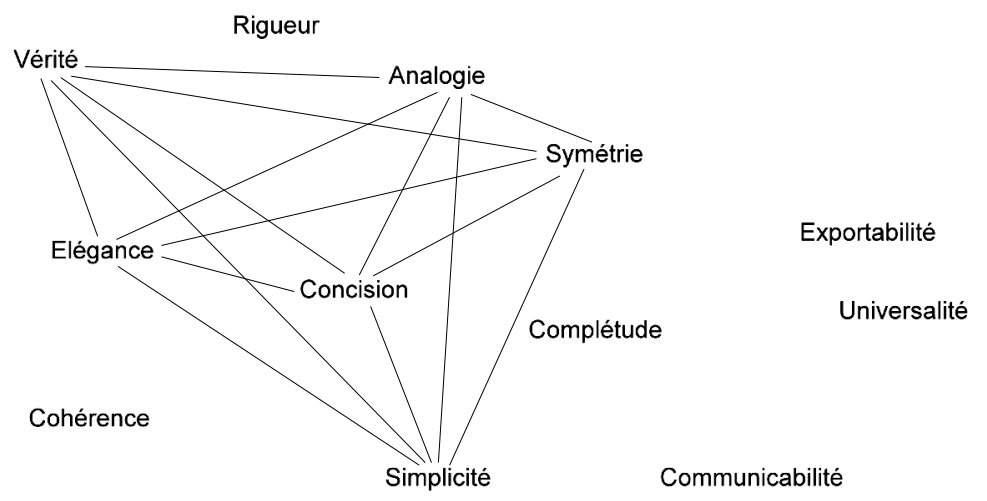

Simplexe esthétique

On remarquera que les arêtes, qui signifient ici l'insertion d'un couple de concepts dans un simplexe, sont toutes de la même nature ou d'importance égale si l'on veut. Cela correspond à la simplification inhérente à notre approche. Dans un modèle plus détaillé on pourrait leur attribuer des valeurs différentes, et pondérer ainsi l'importance de la présence de ce couple dans le simplexe.

Bien sûr il y a d'autres marques d'arbitraire dans la construction de ce simplexe, qui ne reflète qu'une vision personnelle de la situation. Cet arbitraire s'exprime tout d'abord dans le choix - discutable - des concepts, puis dans la construction des simplexes. L'existence des arêtes est le résultat de considérations personnelles. Mais le simplexe permet de se faire une représentation topologique, c'est-à-dire purement situationnelle, de la manière dont les notions évoquées sont connectées dans le contexte de l'orientation émotionnelle mathématique.

Il est intéressant de comparer ce premier simplexe avec le suivant, qui reflète l'aspect «action» du processus mathématique. Ce simplexe est de dimension inférieure et relie des concepts dont l'importance se révèle dans la question de l'efficacité des mathématiques.

Le simplexe opérationnel est de dimension quatre, donc il fait intervenir un plus petit nombre de concepts que le simplexe esthétique, auquel il est attaché autour de l'axe vérité - concision. Il décrit l'aspect calculatoire et les exigences que la raison esthétique impose à la démarche mathématique pour atteindre son efficacité opérationnelle.

Quant au simplexe social, qui illustre la coloration sociale de l'orientation émotionnelle mathématique, il est également de dimension quatre, rattaché au simplexe opérationnel par le seul concept de concision, alors qu'il possède l'axe concision-simplicité en commun avec le simplexe esthétique. Les aspects esthétique et social ne sont donc pas indépendants et démentent la réputation d'activité solitaire qui reste attachée à la démarche mathématique, du moins en ce qui 


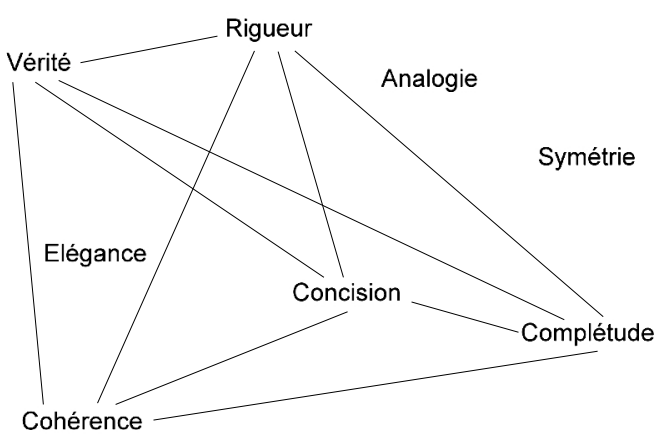

Exportabilité

Universalité

Simplicité

Communicabilité

\section{Simplexe opérationnel}

concerne la recherche. Voici une représentation topologique de cette facette sociale. On y trouve liés, les concepts qui montrent que l'activité mathématique est partagée par une communauté et ne ressemble en rien à une démarche isolée.

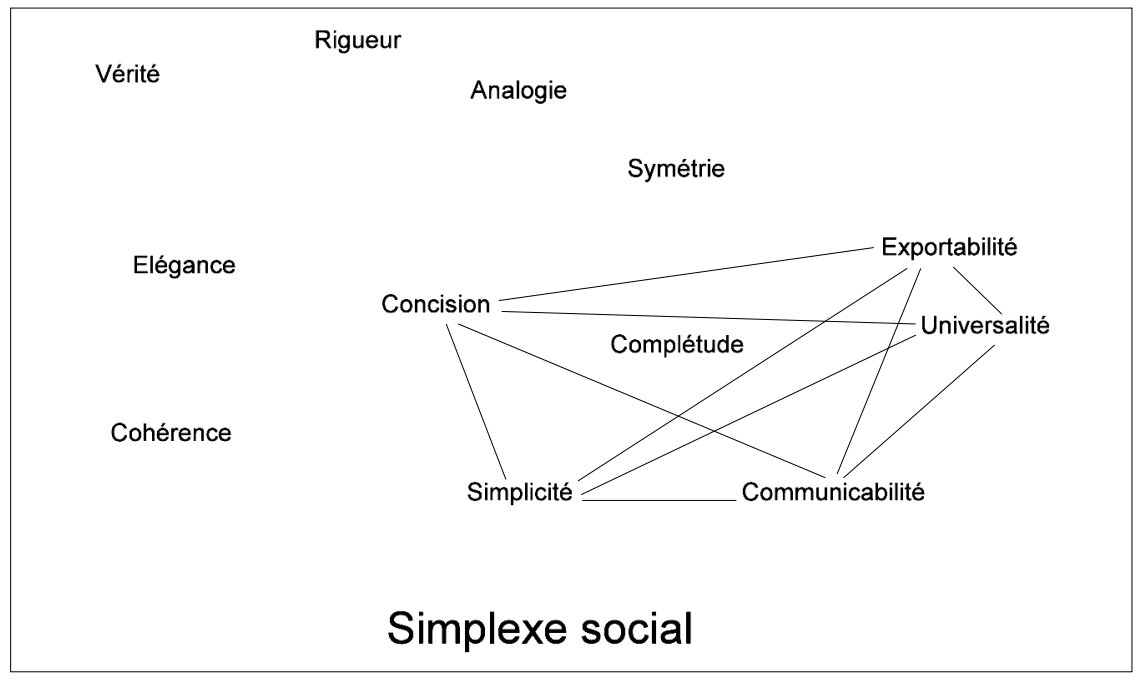

Ce simplexe fait apparaître clairement ce qui vient d'être dit, c'est-à-dire l'importance des considérations sociales, souvent ignorées ou minimisées, qui font du travail du mathématicien non pas une activité solitaire, mais montrent la nécessité pour lui de communiquer, d'échanger et de partager un savoir, des questions et des conventions avec une communauté plus large. 


\section{EN GUISE DE CONCLUSION}

Nous avons considéré dans ces pages l'orientation émotionnelle du mathématicien comme un assemblage indissociable ${ }^{16}$ de croyances, d'expériences, de valeurs partagées, et de manières de communiquer et d'agir dans le cadre d'un groupe. Il n'y a pas, dans cette façon de voir, de séparation nette entre cognition et émotion, les deux axes étant intégrés dans un complexe plus étendu. Cette intégration peut être précisée en distinguant plusieurs facettes, les simplexes, et en étudiant - nous ne l'avons fait ici que très brièvement - leurs parentés, leurs différences et leurs interactions.

La raison esthétique est l'un des moteurs essentiels de la création mathématique. Nous lui avons consacré une place particulièrement importante parce que ses composantes affectives sont souvent cachées, même si le discours spontané des mathématiciens les révèle clairement, comme le montrent les quelques citations proposées.

Emotion - en particulier esthétique - et partage social font donc intégralement partie de la démarche mathématique, contrairement à la réputation dont cette discipline est affublée, et qui tente d'en faire une activité élitiste, solitaire et froide. Il s'agit au contraire d'un domaine où l'implication affective est forte et forme avec le processus de cognition un ensemble complexe, l'orientation émotionnelle mathématique.

Institut de Mathématiques Appliquées, Université de Lausanne

${ }^{16}$ Pour Piaget les fonctions affectives et cognitives étaient de nature différente, mais indissociables selon ses propres termes. Cf J. Piaget, Les relations entre l'affectivité et l'intelligence chez l'enfant, 1954. 